\title{
NMR relaxation rates for the spin-1/2 Heisenberg chain
}

\author{
Anders W. Sandvik因 \\ National High Magnetic Field Laboratory, Florida State University, 1800 E. Paul Dirac Dr., \\ Tallahassee, Florida 32306
}

\begin{abstract}
The spin-lattice relaxation rate $1 / T_{1}$ and the spin echo decay rate $1 / T_{2 G}$ for the spin- $\frac{1}{2}$ antiferromagnetic Heisenberg chain are calculated using quantum Monte Carlo and maximum entropy analytic continuation. The results are compared with recent analytical calculations by Sachdev. If the nuclear hyperfine form factor $A_{q}$ is strongly peaked around $q=\pi$ the predicted lowtemperature behavior $\left[1 / T_{1} \sim \ln ^{1 / 2}(1 / T), 1 / T_{2 G} \sim \ln ^{1 / 2}(1 / T) / \sqrt{T}\right]$ extends up to temperatures as high as $T / J \approx 0.5$. If $A_{q}$ has significant weight for $q \approx 0$ there are large contributions from diffusive long-wavelength processes not taken into account in the theory, and very low temperatures are needed in order to observe the asymptotic $T \rightarrow 0$ forms.
\end{abstract}


The one-dimensional spin- $\frac{1}{2}$ antiferromagnetic Heisenberg hamiltonian,

$$
\hat{H}=J \sum_{i} \vec{S}_{i} \cdot \vec{S}_{i+1}
$$

is relevant as a starting point for understanding the magnetic properties of many quasi one-dimensional materials. Examples include $\mathrm{CuCl} \cdot 2 \mathrm{~N}\left(\mathrm{C}_{5} \mathrm{H}_{5}\right), 1 \mathrm{KCuF}_{3}, 2$ and several tetracyanoquinodimethan (TCNQ) charge transfer salts 3 细 NMR and NQR are commonly used techniques for studying the spin dynamics of materials such as those listed above. The lowfrequency dynamic susceptibility is accessible through the spin-lattice relaxation rate $1 / T_{1}$ and the spin echo decay rate $1 / T_{2 G}$. Theoretical results for the temperature dependence of both these rates were recently obtained by Sachdev 5 using a form for the dynamic susceptibility first derived by Schulz $\mathbf{6}^{6}$ using the Bosonization method. Neglecting logarithmic corrections, $1 / T_{1}$ is predicted to be constant at low temperature, and $1 / T_{2 G}$ is predicted to diverge as $T^{-1 / 2}$. With logarithmic corrections taken into account both rates acquire a factor $\ln ^{1 / 2}(1 / T)$. These results are expected to be valid only for temperatures $T \ll J$, and it is important to verify their validity as well as to obtain results also at higher temperatures. Here results are presented for $1 / T_{1}$ and $1 / T_{2 G}$ computed using quantum Monte Carlo (QMC) simulations of chains of up to 1024 spins. $1 / T_{2 G}$ is related to static susceptibilities directly computable in the simulations. The dynamic susceptibility required for extracting $1 / T_{1}$ is calculated in imaginary time and continued to real frequency using the maximum entropy method.7.

The results for the temperature dependence of both $1 / T_{1}$ and $1 / T_{2 G}$ at low temperatures are in good agreement with Sachdev's predictions. At higher temperatures diffusive modes not taken into account in the theory cause significant deviations. If the nuclear hyperfine form factor has large weight at long wavelengths very low temperatures are needed for the asymptotic forms to apply, and they may then be difficult to observe experimentally. The results presented here should be useful for comparisons with experiments also at higher temperatures.

The NMR spin-lattice relaxation rate is given by 


$$
\frac{1}{T_{1}}=\frac{1}{N} \sum_{\alpha} \sum_{q}\left|A_{q}^{\alpha}\right|^{2} S(q, \omega \rightarrow 0),
$$

where $A_{q}^{\alpha}$ is the hyperfine form factor, and $\alpha$ denotes the two axes perpendicular to the external field direction. $S(q, \omega)$ is the dynamic structure factor, which is related to the imaginary part of the dynamic spin susceptibility according to $S(q, \omega)=\chi^{\prime \prime}(q, \omega) /\left(1-\mathrm{e}^{-\beta \omega}\right)$, where $\beta=1 / k_{B} T$. Here an isotropic form factor $A_{q}^{\alpha}=A_{q}$ will be assumed. Defining

$$
S_{A}(\omega)=\frac{1}{N} \sum_{q}\left|A_{q}\right|^{2} S(q, \omega)
$$

the spin-lattice relaxation rate is then obtained as $1 / T_{1}=2 S_{A}(\omega \rightarrow 0)$. For the numerical calculations carried out here it is more convenient to work directly with the hyperfine coupling $A(r)$ in coordinate space. Define

$$
C_{A}(\tau)=\sum_{i, j} A\left(r_{i}\right) A\left(r_{j}\right)\left\langle S_{i}^{z}(\tau) S_{j}^{z}(0)\right\rangle
$$

where $S_{i}^{z}(\tau)=\mathrm{e}^{\tau \hat{H}} S_{i}^{z} \mathrm{e}^{-\tau H}$. With $C_{A}(\tau)$ calculated numerically, $S_{A}(\omega)$ can be obtained by inverting the relation

$$
C_{A}(\tau)=\frac{1}{\pi} \int_{-\infty}^{\infty} d \omega S_{A}(\omega) \mathrm{e}^{-\tau \omega}
$$

using the maximum entropy technique. 영 This method is described in detail in Ref. 8, and was recently applied in a calculation of the spin-lattice relaxation rate of the two-dimensional Heisenberg model. 10

The gaussian component of the spin echo decay rate is related to the the nuclear spinspin interactions mediated by the electrons. Under conditions discussed by Pennington and Slichter 11

$$
\frac{1}{T_{2 G}}=\left[\frac{1}{2} \sum_{x \neq 0} J_{z}^{2}(0, x)\right]^{1 / 2}
$$

where $J_{z}\left(x_{1}, x_{2}\right)$ is the $z$-component of the induced interaction between nuclei at $x_{1}$ and $x_{2}$ :

$$
J_{z}\left(x_{1}, x_{2}\right)=-\frac{1}{2} \sum_{i, j} A\left(x_{1}-r_{i}\right) A\left(x_{2}-r_{j}\right) \chi(i-j)
$$


The static susceptibility $\chi(i-j)$ is given by the Kubo formula

$$
\chi(i-j)=\int_{0}^{\beta} d \tau\left\langle S_{i}^{z}(\tau) S_{j}^{z}(0)\right\rangle .
$$

The hyperfine interaction $A(r)$ is normally very short ranged. Here a situation is considered where the nuclei studied reside at the sites of the electronic spins modeled by the hamiltonian (11). The hyperfine coupling is assumed to have a direct contact term of strength $A(0)$, and a transferred nearest-neighbor term of strength $A(1)$. Results are presented for several values of the ratio $R=A(1) / A(0)$.

A stable inversion of the relation (5) requires that $C_{A}(\tau)$ is known to very high accuracy. Here a quantum Monte Carlo method based on stochastic series expansion 12 (a generalization of Handscomb's method 13 ) is used. This technique is free from systematical errors of the "Trotter break-up" used in standard methods.14 The imaginary time correlation functions needed have been calculated to within relative statistical errors of only $10^{-4}$ or lower for temperatures down to $T=J / 8$. This high accuracy is required for obtaining a reliable estimate of $S_{A}(\omega \rightarrow 0)$. The static susceptibilities (8) are computed directly in the QMC simulation, and hence the calculation of $1 / T_{2 G}$ is not hampered by potential problems associated with analytic continuation. Accurate results for $1 / T_{2 G}$ have been obtained at temperatures as low as $T=J / 32$ for systems of up to 1024 sites, which is large enough for finite-size effects to be completely negligible.

In order to test the accuracy of a calculation of $1 / T_{1}$ by analytic continuation of QMC data, complete diagonalizations of 16 -site chains were also carried out. Comparisons of $S(\omega)=\frac{1}{N} \sum_{q} S(q, \omega)$ obtained in these calculations with numerically continued QMC data are shown in Fig. 1. The maximum entropy method requires a "default model" which defines the zero of entropy. diagonalization gives $S(\omega)$ as a finite number of delta functions and their corresponding weights. Here the results are plotted as histograms in order to facilitate comparison with the maximum entropy result. The jagged structure of the exact diagonalization result, which is due to the small size of the system, cannot be reproduced by the maximum entropy 
method. The results do however represent reasonable frequency averages. Note that even the high-frequency behavior is obtained quite accurately. Clearly a 16-site system is not large enough for extracting the low-frequency behavior at low temperatures. The $1 / T_{1}$ results presented below are for systems of 256 spins, and the accuracy of the imaginary time data used for the analytic continuation is even higher than the data used for the 16-site results shown in Fig. 1. Comparisons with results obtained for 128 spins indicate that there are no significant finite size effects at the temperatures considered.

Both fluctuations of the uniform $(q \approx 0)$ and staggered $(q \approx \pi)$ magnetization contribute to the NMR rates of half-integer spin chains. At low temperatures the staggered contribution dominates 5 Neglecting the uniform fluctuations, Sachdev obtained the asymptotic low-temperature forms (in units where $\hbar=k_{B}=1$ )

$$
\begin{aligned}
\frac{1}{T_{1}} & =A_{\pi}^{2} \frac{\pi D}{c}, \\
\frac{1}{T_{2 G}} & =A_{\pi}^{2} \frac{I D}{4} \sqrt{\frac{1}{2 c T}},
\end{aligned}
$$

where $I \approx 8.4425$, and $c$ is the spinon velocity, which for spin- $\frac{1}{2}$ is $c=\frac{\pi}{2} . D$ is the prefactor of the asymptotic equal-time spin-correlation function, which is not known accurately. 1 . The marginally irrelevant operator present for the critical spin chains has not been taken into account in the derivation of the above forms. This is expected to lead to a multiplicative correction $\ln { }^{1 / 2}(\Lambda / T)$ for both $1 / T_{1}$ and $1 / T_{2 G}$. 国 Hence, the ratio $T_{2 G} /\left(\sqrt{T} T_{1}\right)$ should be a constant, even with logarithmic corrections included.

If $1 / T_{2 G} \sim \ln ^{1 / 2}(\Lambda / T) / \sqrt{T}$ as predicted by Sachdev, $T\left(1 / T_{2 G}\right)^{2}$ should be a linear function of $\ln (J / T)$. In Fig. $2, T\left(A_{\pi}^{2} T_{2 G}\right)^{-2}$ is graphed versus $\ln (J / T)$ for several values of the hyperfine coupling ratio $R=A(1) / A(0)$. In cases where the corresponding $A_{q}$ is peaked around $q=\pi(R<0)$ a linear behavior is seen in a wide temperature regime. The points for $R=-0.25$ and $R=-0.5$ nearly coincide at low $T$, indicating that the $q \approx \pi$ contributions almost completely dominate the behavior in both cases. A line fit to the $R=-0.5$ points gives $\Lambda=0.92 J$ and the amplitude $D=0.080$ in (9D). For $R>0$ contributions from $q \approx 0$ rapidly become important at high temperatures, and for large values of $R$ the 
asymptotic behavior can only be observed at very low temperatures.

Results for $1 / T_{1}$ divided by $A_{\pi}^{2}$ are shown in Fig. 3. The expected weak (logarithmic) increase as $T$ decreases can be seen below $T / J=0.5$ if $R$ is large and negative, so that $q \approx \pi$ processes dominate $S_{A}(\omega \rightarrow 0)$ even at relatively high temperatures. For $R>0,1 / T_{1}$ decreases with decreasing $T$ down to quite low temperatures - for $R=0.25$ this behavior extends down to the lowest temperature studied. The enhancement of $1 / T_{1}$ at high $T$ is caused by the diffusive $q \approx 0$ processes not taken into account in the forms (9). more clearly determine the importance of these modes one can study the ratio

$$
\frac{S_{q<\pi / 2}(\omega \rightarrow 0)}{S(\omega \rightarrow 0)}=\frac{\sum_{q<\pi / 2} S(q, \omega \rightarrow 0)}{\sum_{q} S(q, \omega \rightarrow 0)},
$$

which is graphed versus the temperature in Fig. 4 (these calculations were carried out on systems of 128 spins). At $T=J$ the $q<\pi / 2$ contribution is approximately $50 \%$, and decreases rapidly at lower temperatures. These results confirm Sachdev's conclusionl that the $q \approx 0$ contribution to $1 / T_{1}$ is negligible in the limit $T \rightarrow 0$.

Returning now to the results shown in Fig. 3, there are not enough low-temperature data to extract the asymptotic temperature dependence of $1 / T_{1}$. The results are, however, consistent with a divergence of the predicted form $\ln ^{1 / 2}(\Lambda / T)$ with the same $\Lambda=0.92 J$ as was found above for $1 / T_{2 G}$. The amplitude needed in Eq. (9b) is then $D \approx 0.14$, which is significantly larger than the amplitude extracted from $1 / T_{2 G}$ above. Hence, the ratio $T_{2 G} /\left(\sqrt{T} T_{1}\right)$ is different from Sachdev's prediction. The ratio is graphed versus temperature in Fig. 5. For $R<0$ it is indeed almost constant below $T / J \approx 0.5$, whereas for $R \geq 0$ there is a significant temperature dependence down to the lowest temperatures considered. For positive $R$ there is a sharp maximum in $T_{2 G} /\left(\sqrt{T} T_{1}\right)$, arising from the minimum in $1 / T_{2 G}$ seen in Fig. 2. The $R=-0.5$ result for $T_{2 G} /\left(\sqrt{T} T_{1}\right)$ at low temperatures is approximately $3.0-3.1$, which is almost a factor 2 larger than what is obtained from Eqs. (9).

In summary, the NMR rates $1 / T_{1}$ and $1 / T_{2 G}$ have been calculated for the spin- $1 / 2$ Heisenberg model, using quantum Monte Carlo and maximum entropy analytic continuation. The temperature dependence at low temperature is in good agreement with Sachdev's 
recent theoretical results, which include only the contributions from staggered magnetization fluctuations. At high temperature damped $q \approx 0$ modes are important, and can dominate the NMR rates if the hyperfine form factor has large weight at long wavelengths. In such cases very low temperatures are needed to observe the asymptotic forms. In many real systems effects of interchain couplings may become important before the asymptotic regime is reached, and the low-temperature forms may therefore not be easily observed. The results here should then be useful for determining the relevance of a description by the one-dimensional Heisenberg model based upon measurements at higher temperatures. It can be noted that early NMR experiments on (NMP)(TCNQ) indicate a behavior of $1 / T_{1}$ similar to the result shown in Fig. 3 for a small positive hyperfine ratio $A(1) / A(0)$, with no indication of a low-temperature increase down to $T \approx 0.1 J$.

It will be interesting to apply the techniques used here to calculate the NMR rates of other one-dimensional systems. Work on coupled spin chains is in progress 16 Itinerant electrons described by one-dimensional Hubbard-type models, including electron-phonon interactions, can also be studied.

I would like to thank E. Dagotto, S. Haas, and D. Scalapino for useful discussions. Most of the computations were carried out on a cluster of DEC Alpha workstations at the Supercomputer Computations Research Institute at Florida State University. This work was supported by the Office of Naval Research under Grant No. ONR N00014-93-0495. 


\section{REFERENCES}

* On leave from Department of Physics. Åbo Akademi University, Åbo, Finland.

${ }^{1}$ Y. Endoh et al. Phys. Rev. Lett. 32, 170 (1974).

${ }^{2}$ D. A. Tennant et al., Phys. Rev. Lett. 70403 (1993).

${ }^{3}$ A. J. Epstein et al., Phys. Rev. B5, 952 (1971).

${ }^{4}$ E. Ehrenfreund et al., Phys. Rev. B7, 421 (1973).

${ }^{5}$ S. Sachdev, Phys. Rev. B50, 13006 (1994).

${ }^{6}$ H. J. Schulz, Phys. Rev. B34, 6372 (1986).

${ }^{7}$ J. E. Gubernatis et al., Phys. Rev. B44 (1991) 6011.

${ }^{8}$ M. Jarrell and J. E. Gubernatis (to appear in Phys. Rep.).

${ }^{9}$ T. Moriya, Prog. Theor. Phys. 28, 371 (1962).

${ }^{10}$ A. W. Sandvik and D. J. Scalapino, Phys. Rev. B51, 9403 (1995).

${ }^{11}$ C. H. Pennington and C. P. Slichter, Phys. Rev. Lett. 66, 381 (1991).

${ }^{12}$ A. W. Sandvik and J. Kurkijärvi, Phys. Rev. B43, 5950 (1991); A. W. Sandvik, J. Phys. A25, 3667 (1992).

${ }^{13}$ D. C. Handscomb, Proc. Cambridge Philos. Soc. 58, 594 (1962); 60, 116 (1964).

${ }^{14}$ J. E. Hirsch et al., Phys. Rev. B 26, 5033 (1982).

${ }^{15}$ For a recent numerical estimate of D, see K. Hallberg, P. Horsch, and G. Martinez, Phys. Rev. B 52, R719 (1995).

${ }^{16}$ A. W. Sandvik, E. Dagotto, and D. J. Scalapino (unpublished). 


\section{FIGURES}

FIG. 1. QMC and exact diagonalization results for $S(\omega)=\frac{1}{N} \sum_{q} S(q, \omega)$ of a 16-site chain. The exact results are plotted as histograms, and the curves are numerically continued QMC imaginary time correlation functions.

FIG. 2. Results for the spin echo decay rate, graphed as $T\left(A_{\pi}^{2} T_{2 G}\right)^{-2}$ vs. $\ln (J / T)$ for different hyperfine ratios $R=A(1) / A(0)$.

FIG. 3. Spin-lattice relaxation rates vs. temperature for different hyperfine ratios $R=$ $A(1) / A(0)$. The solid curve is of the form $\ln (\Lambda / T)$, with $\Lambda=0.92 J$.

FIG. 4. The long-wavelength contribution to $S(\omega \rightarrow 0)=\sum_{q} S(q, \omega \rightarrow 0)$ vs. temperature.

FIG. 5. The ratio $T_{2 G} / \sqrt{T} T_{1}$ vs. $T$ for different hyperfine ratios $R=A(1) / A(0)$. 

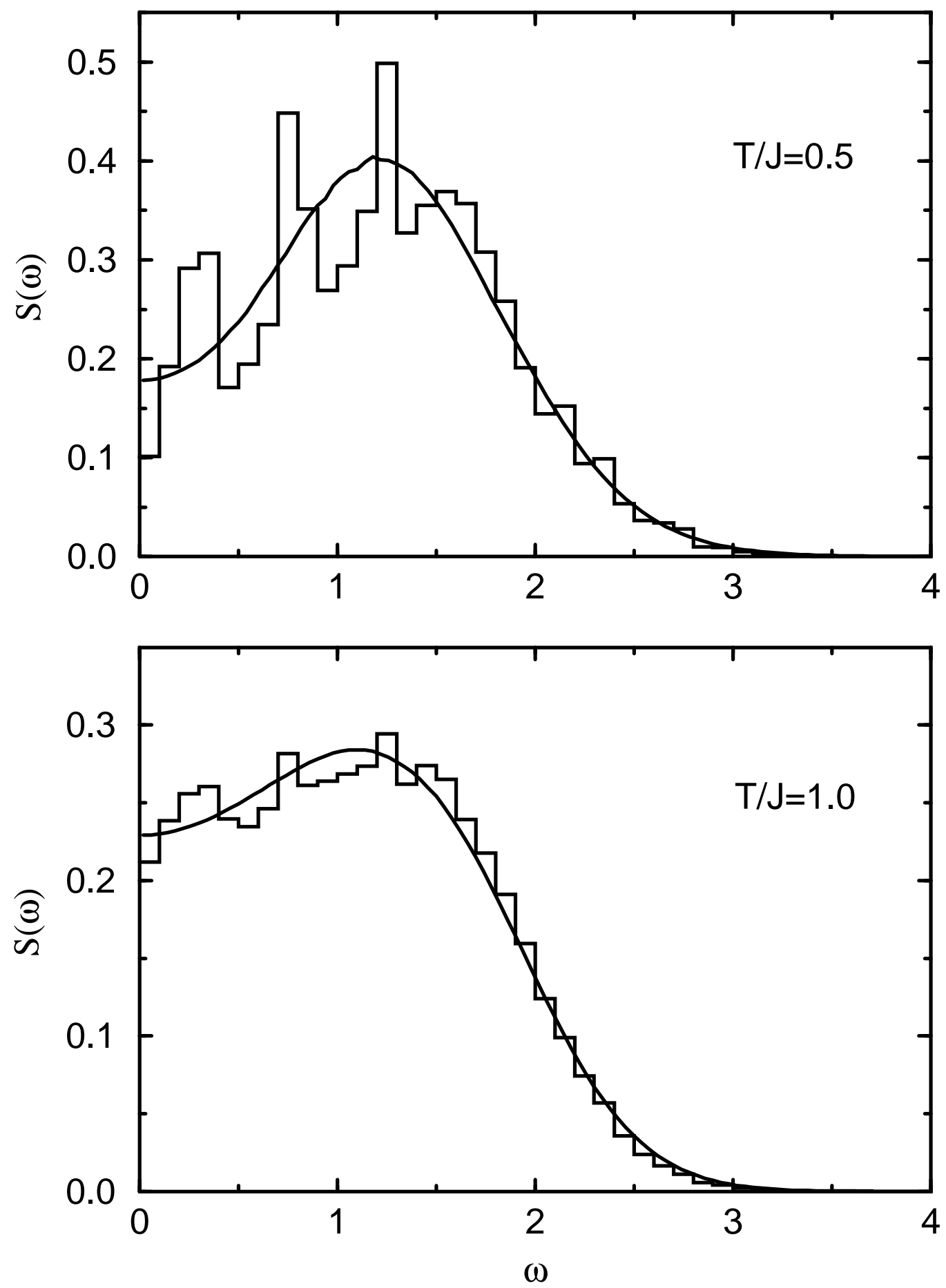

FIG. 1, A. W. Sandvik 


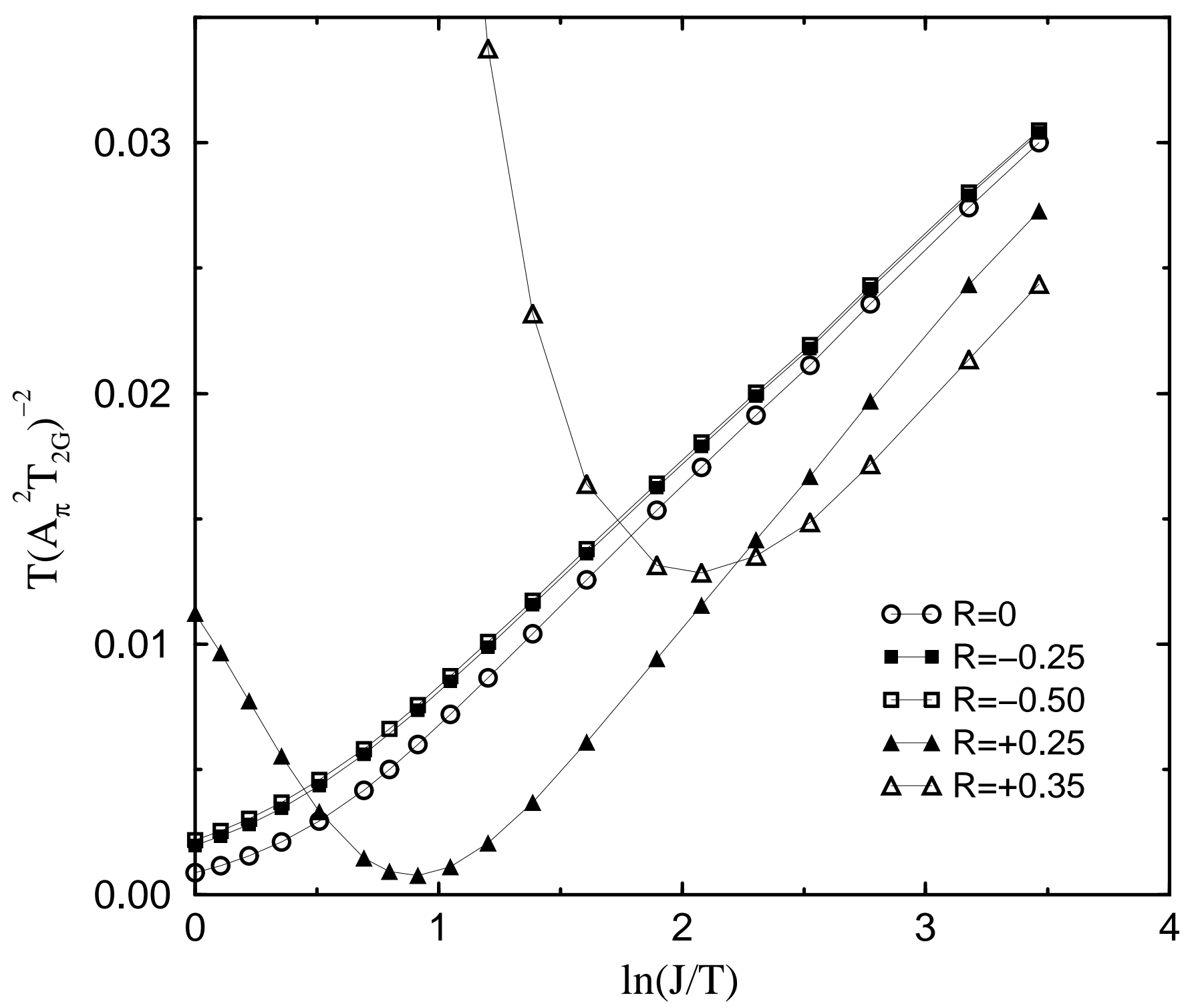

FIG. 2, A. W. Sandvik 


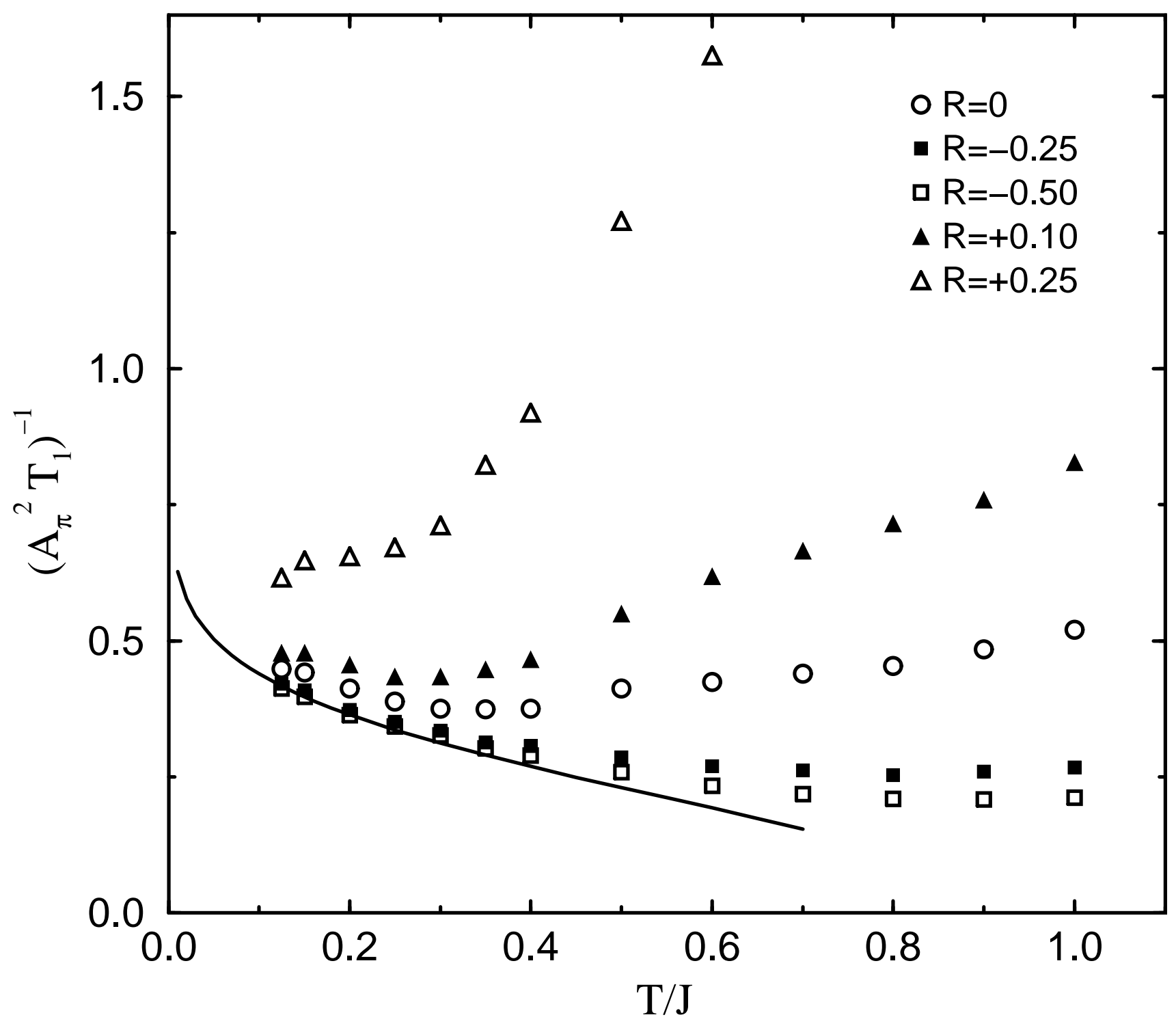

FIG. 3, A. W. Sandvik 


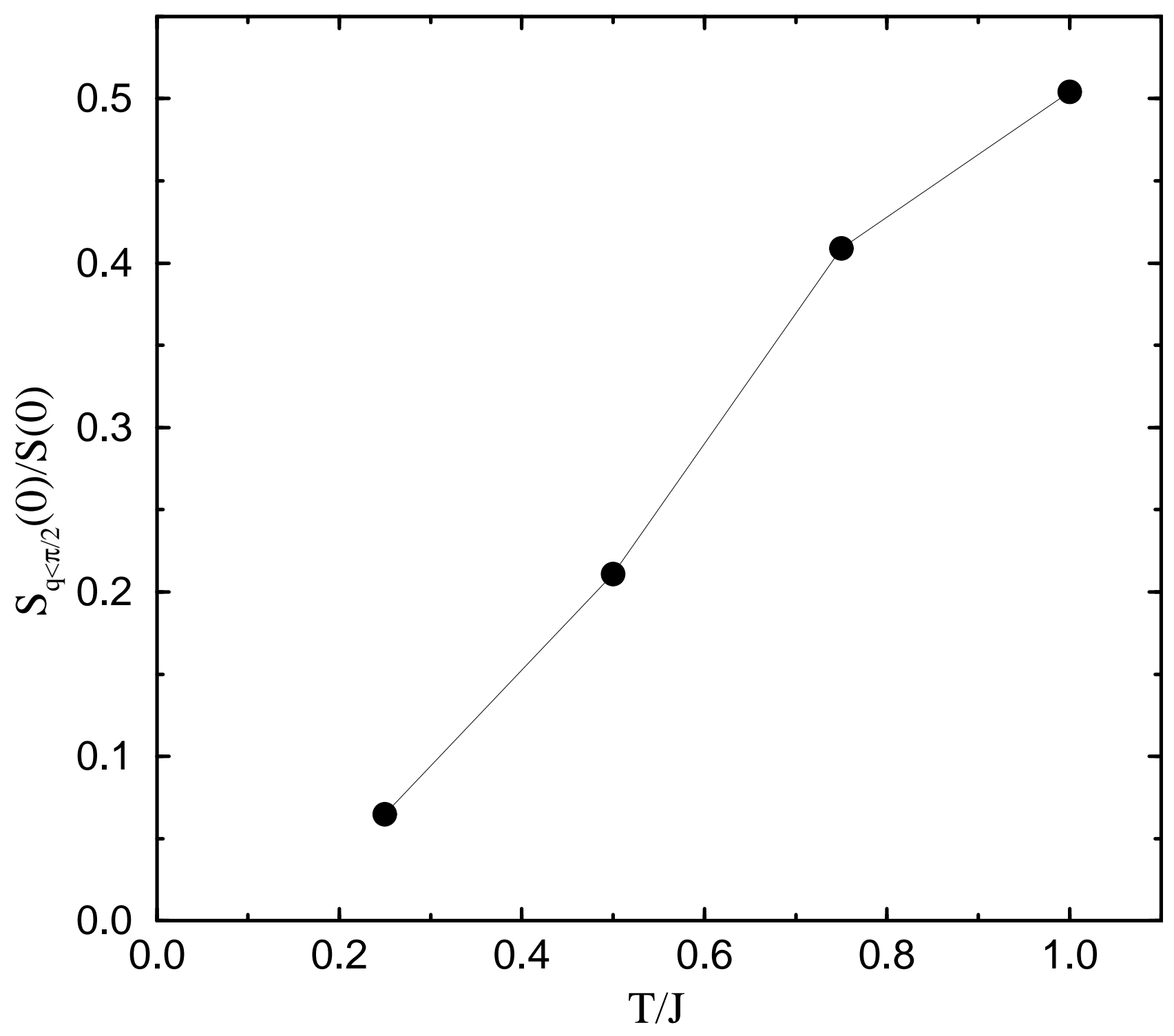

FIG. 4, A. W. Sandvik 


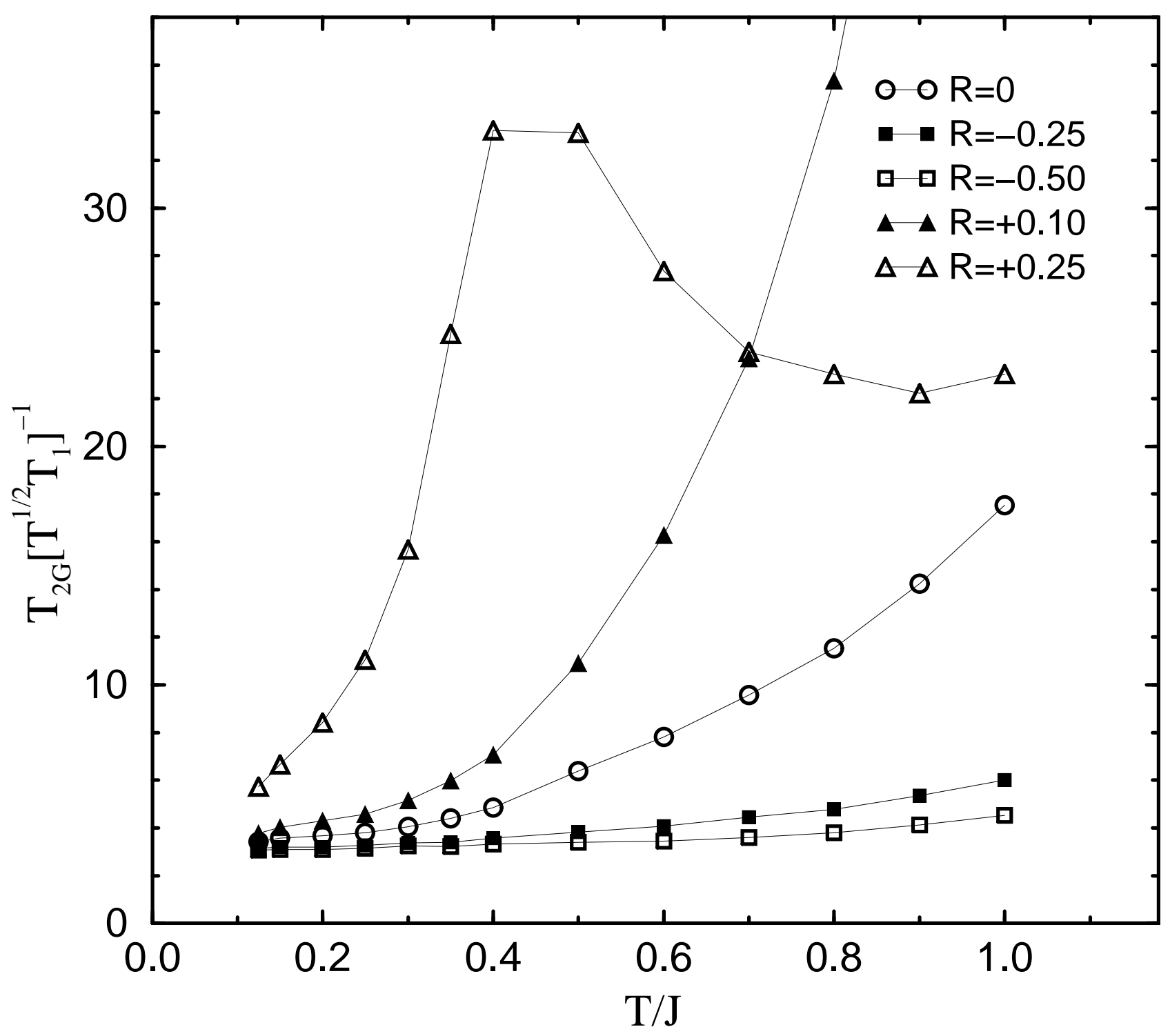

FIG. 5, A. W. Sandvik 